\title{
Ácaros plumícolas em aves Passeriformes da Zona da Mata Norte de Pernambuco, Brasil
}

\author{
Sônia Aline Roda ${ }^{1}$ \\ Ângela Maria Isidro de Farias ${ }^{1}$
}

\begin{abstract}
Feather mites on Passeriformes birds from North Zona da Mata, Pernambuco, Brazil. Studies were carried out on the relationship between feather mites in wild birds captured by mist nets at the Engenho Independência, county of Vicência. The captures were performed in June, September and December (1995) and March (1996). Mites belonging to the families: Analgidae, Psoroptoididae, Avenzoariidae, Proctophyllodidae e Trouessartiidae were identified to genera. The most representative genera, according to the number of hosts they were found were Pterodectes sp. (Proctophyllodidae), with eighteen and Trouessartia sp. (Trouessartiidae), with sixteen hosts, respectively. The Psoroptoididae and Avenzoariidae families were found in only one host each. The ventral face of remiges and rectrices were the main sites inhabited by mites.

KEY WORDS. Ectoparasites, Acari, feather mites, wild birds, Pernambuco, host-parasite relationship
\end{abstract}

As aves silvestres abrigam uma fauna de ectoparasitos típica. Segundo GAUD \& ATYEO (1979), cada ordem da classe Aves apresenta uma acarofauna definida, evidenciando uma íntima adaptação ao seu hospedeiro. Estes autores citam ainda que a evolução dos ácaros plumícolas possa ter ocorrido paralelamente à das aves, ocorrendo nas penas de contorno, cauda, asas e no interior do cálamo das penas de vôo. Deve-se destacar entretanto, que há ácaros que não apresentam especificidade muito acentuada, como alguns que parasitam aves das famílias Anatidae, Indicatoridae, Cuculidae, Icteridae e Ploceidae (ATYEO \& GAUD 1983).

Os ácaros plumícolas apresentam variações morfológicas de acordo com a sua localização nas penas do hospedeiro. Estes são transferidos para hospedeiros da mesma espécie, em regra, dos pais para os filhos, ainda no ninho (ATYEO \& GAUD 1983; GAUD \& ATYEO 1979).

No Brasil, os escassos estudos sobre ectoparasitos de aves silvestres abordam aspectos das relações parasito-hospedeiro e/ou sistemática dos ácaros plumícolas (BERLA 1958a,b, 1959a,b,c,d, 1960a,b, 1962; FACCINI et al. 1976; FLECHTMANN 1981; Novaes \& CARVALHo 1952a,b; NovaES 1953). São poucos os trabalhos recentes (MARINI et al. 1996; MARINI \& COUTO 1997), e em particular, no nordeste do Brasil.

1) Departamento de Zoologia, Centro de Ciências Biológicas, Departamento de Zoologia, Universidade Federal de Pernambuco. Avenida Professor Morais Rego 1235, Cidade Universitária, 50670-420 Recife, Pernambuco, Brasil.

E-mail: saroda@hotlink.com.br 
Este trabalho tem como principal objetivo identificar os ácaros plumícolas das aves silvestres capturadas na Zona da Mata Norte, Pernambuco e verificar os locais preferenciais desses ectoparasitos em seus respectivos hospedeiros.

\section{MATERIAL E MÉTODOS}

As aves foram capturadas com redes de neblina no Engenho Independência (35⒉ 'W e $7^{\circ} 39^{\prime}$ 'S), situado no município de Vicência, Zona da Mata Norte de Pernambuco, em quatro períodos: junho, setembro e dezembro de 1995 e em março de 1996.

Os ácaros visíveis eram retirados do hospedeiro juntamente com as penas. Estas amostras eram transferidas para frascos de vidro, devidamente individualizados e numerados de acordo com a ficha de campo da ave hospedeira, separados por região do corpo da ave, e em seguida fixadas em álcool a $70 \%$.

No Laboratório de Invertebrados Terrestres do Departamento de Zoologia da Universidade Federal de Pernambuco procedeu-se a preparação, montagem e identificação do material.

As penas retiradas dos hospedeiros, foram observadas sob microscópio estereoscópio em placas de Petri contendo álcool a 70\%. Inicialmente observou-se a disposição dos ácaros nas penas, em seguida estes eram retirados e montados entre lâmina e lamínula, seguindo-se a técnica padronizada em acarologia por FLECHTMANN (1975).

Os ácaros foram identificados com auxílio de literatura especializada. Para a separação das famílias, utilizou-se a classificação de GAUD \& ATYEO (1977).

A infestação por ácaros, para cada espécie de ave hospedeira, foi quantificada usando-se a prevalência, segundo MARGOLIS et al. (1982). Diferenças entre os locais parasitados pelos diferentes gêneros foram avaliadas através da análise de variância, segundo o método de Kruskall-Wallis, sendo as médias comparadas pelo teste de comparação múltipla de Kruskall-Wallis. Todas as análises estatísticas foram realizados a um nível de significância de 5\%.

\section{RESULTADOS E DISCUSSÃO}

As famílias de ácaros e seus respectivos gêneros identificados foram: Analgidae Mégnin \& Trouessart, 1883, Psoroptoididae Gaud, 1980 (Mesalgoides Gaud \& Atyeo, 1967), Avenzoariidae Oudemans, 1905 (Pteronyssoides Hull, 1931), Proctophyllodidae Mégnin \& Trouessart, 1883 (Proctophyllodes Robin, 1868 e Pterodectes Robin, 1877) e Trouessartiidae Gaud, 1957 (Trouessartia Canestrini, 1889).

Indivíduos da família Analgidae, foram encontrados em três hospedeiros: Certhiaxis cinnamomea (Cory, 1916) (Furnariidae), Phacellodomus rufifrons Hellmayr, 1925 (Furnariidae) e Volatinia jacarina (Linnaeus, 1758) (Emberizidae) (Tab. I). Esta família parasita tanto aves Passeriformes, como não Passeriformes (GAUD \& ATYEO 1996). Os espécimens ocorreram em locais diferentes nos três hospedeiros (Tab. I).

O gênero Mesalgoides (Psoroptoididae) foi assinalado apenas em $V$. jacarina (Tab. I). 
Tabela I. Prevalência e locais parasitados por ácaros plumicolas em aves silvestres capturadas no Engenho Independência, Vicência, Zona da Mata Norte de Pernambuco. (FV) Face ventral da pena, (FD) face dorsal da pena, (RM) rêmiges, (RT) retrizes, (CA) cabeça, (URO) uropígeo, (SC) supra-caudais, $(n)$ número de individuos capturados, $(\mathrm{N})$ número de indivíduos parasitados

\begin{tabular}{|c|c|c|c|c|c|}
\hline Hospedeiro & $\mathrm{n}$ & $\mathrm{N}$ & Acari & $\begin{array}{c}\text { Local } \\
\text { Ectoparasitado }\end{array}$ & $\begin{array}{c}\text { Prevalência } \\
\qquad(\%)\end{array}$ \\
\hline \multicolumn{6}{|l|}{ Furnariidae } \\
\hline \multirow[t]{2}{*}{ Furnarius figulus (Lichtenstein, 1823) } & 2 & 2 & Pterodectes sp. & FV e FD RM e RT & 100,00 \\
\hline & 2 & 1 & Trouessartia sp. & FV RM & 50,00 \\
\hline Synallaxis frontalis Pelzeln, 1859 & 1 & 1 & Pterodectes sp. & FV RM & 100,00 \\
\hline \multirow[t]{4}{*}{ Certhiaxis cinnamomea (Cory, 1916) } & 7 & 1 & Analgidae & CA & 14,30 \\
\hline & 7 & 2 & Proctophyllodes sp. & FVRM e RT & 28,50 \\
\hline & 7 & 1 & Pterodectes sp. & FV e FD RT & 14,30 \\
\hline & 7 & 6 & Trouessartia sp. & FV RM & 85,70 \\
\hline \multirow[t]{3}{*}{ Phacellodomus rufifrons Hellmayr, 1925} & 9 & 1 & Analgidae & SC & 11,10 \\
\hline & 9 & 3 & Pterodectes sp. & FV RM, FV e FD RT & 33,30 \\
\hline & 9 & 3 & Trouessartia sp. & FV RM, FD RT & 33,30 \\
\hline \multicolumn{6}{|l|}{ Tyrannidae } \\
\hline \multirow[t]{2}{*}{ Elaenia flavogaster (Thunberg, 1822) } & 16 & 7 & Pterodectes sp. & FV e FD RM e RT & 43,70 \\
\hline & 16 & 4 & Trouessartia sp. & FVRM e RT & 25,00 \\
\hline Hemitriccus mirandae Cabanis \& Heine, 1859 & 1 & 1 & Trouessartia sp. & FVRM & 100,00 \\
\hline \multirow[t]{2}{*}{ Myiophobus fasciatus (Temmink, 1822) } & 2 & 1 & Pterodectes sp. & FV RM & 50,00 \\
\hline & 2 & 2 & Trouessartia sp. & FV e FD RM, FV RT & 100,00 \\
\hline \multirow[t]{3}{*}{ Tyrannus melancholicus (Lichtenstein, 1823) } & 2 & 1 & Proctophyllodes sp. & FV RM e RT & 50,00 \\
\hline & 2 & 2 & Pterodectes sp. & FV RM & 100,00 \\
\hline & 2 & 1 & Trouessartia sp. & FD RT & 50,00 \\
\hline \multicolumn{6}{|l|}{ Hirundinidae } \\
\hline \multirow[t]{4}{*}{ Stelgidopteryx ruficollis (Vieillot, 1817) } & 31 & 17 & Pteronyssoides sp. & FV e FD RM e RT & 54,83 \\
\hline & 31 & 1 & Proctophyllodes sp. & FV RM & 3,20 \\
\hline & 31 & 1 & Pterodectes sp. & FV RM, FV e FD RT & 3,20 \\
\hline & 31 & 18 & Trouessartia sp. & FV e FD RM e RT & 58,00 \\
\hline \multicolumn{6}{|l|}{ Troglodytidae } \\
\hline Troglodytes aedon Naumann, 1823 & 3 & 1 & Trouessartia sp. & FV e FD RM & 33,30 \\
\hline \multicolumn{6}{|l|}{ Muscicapidae } \\
\hline \multirow[t]{3}{*}{ Turdus rufiventris (Cory, 1916) } & 2 & 1 & Proctophyllodes sp. & FV RM e RT & 50,00 \\
\hline & 2 & 2 & Pterodectes sp. & FVe FD RT & 100,00 \\
\hline & 2 & 1 & Trouessartia sp. & FV RM & 50,00 \\
\hline \multicolumn{6}{|l|}{ Emberizidae } \\
\hline \multirow[t]{2}{*}{ Coereba flaveola Vieillot, 1808} & 12 & 10 & Pterodectes sp. & FV RM, FV e FD RT & 83,30 \\
\hline & 12 & 1 & Trouessartia sp. & $\mathrm{CA}$ & 8,30 \\
\hline \multirow[t]{2}{*}{ Thlypopsis sordida Lafresnaye \& d'Orbigny, 1837} & 7 & 4 & Pterodectes sp. & FV RM, FV e FD RT, CA & 57,10 \\
\hline & 7 & 1 & Trouessartia sp. & FD RT & 14,30 \\
\hline \multirow[t]{3}{*}{ Thraupis sayaca (Linnaeus, 1766) } & 7 & 1 & Proctophyllodes sp. & FV RM & 14,30 \\
\hline & 7 & 4 & Pterodectes sp. & FV RM & 57,10 \\
\hline & 7 & 3 & Trouessartia sp. & FD RM & 42,80 \\
\hline \multirow[t]{2}{*}{ Tangara cayana (Gmelin, 1789) } & 5 & 1 & Proctophyllodes sp. & FV RM e RT & 20,00 \\
\hline & 5 & 3 & Pterodectes sp. & FV RM, FV e FD RT & 60,00 \\
\hline \multirow[t]{5}{*}{ Volatinia jacarina (Linnaeus, 1758) } & 4 & 3 & Analgidae & FVRM & 75,00 \\
\hline & 4 & 2 & Mesalgoides sp. & FV RM E RT, URO & 50,00 \\
\hline & 4 & 1 & Proctophyllodes sp. & FDRM & 25,00 \\
\hline & 4 & 3 & Pterodectes sp. & FV RM, FV e FD RT & 75,00 \\
\hline & 4 & 1 & Trouessartia sp. & FD RM e RT & 25,00 \\
\hline Sporophila lineola (Linnaeus, 1766) & 2 & 2 & Pterodectes sp. & FV RM, FV e FD RT, CA & 100,00 \\
\hline Sporophila nigricollis (Vieillot, 1823) & 6 & 1 & Proctophyllodes sp. & FD RT & 16,70 \\
\hline & 6 & 1 & Pterodectes sp. & FV e FD RT & 16,70 \\
\hline & 6 & 2 & Trouessartia sp. & FV RM & 33,33 \\
\hline Sporophila albogularis (Spix, 1825) & 2 & 1 & Pterodectes sp. & FV RM & 50,00 \\
\hline Paroaria dominicana (Linnaeus, 1758) & 2 & 1 & Trouessartia sp. & FV RM & 50,00 \\
\hline Passer domesticus (Linnaeus, 1760) & 6 & 2 & Proctophyllodes sp. & FVRM e RT & 33,30 \\
\hline & 6 & 1 & Pterodectes sp. & FV RM & 16,70 \\
\hline
\end{tabular}


O gênero Pteronyssoides, representante da família Avenzoariidae, esteve presente apenas em Stelgidopteryx ruficollis (Hirundinidae) (Tab. I). Encontrou-se estes ácaros parasitando as rêmiges e retrizes, no entanto, FACCINI \& ATYEO (1981) assinalam que estes ocorrem principalmente nas rêmiges primárias.

FACCINI \& ATYEO (1981) citam a ocorrência de Pteronyssoides para cerca de setenta espécies de aves Passeriformes, distribuídas em muitas famílias. GAUD \& ATYEO (1985) citam seis espécies deste gênero, como ectoparasitos da família Hirundinidae: P. tyrelli (Canestrini, 1882), P. obscurus (Berlese, 1884), P. infuscatus (Trt, 1885), P. nuntiaeveris (Berlese, 1886), P. bifurcatus (Haller, 1912) e $P$. atticorae Cerny, 1975. Estes autores assinalam ainda, que as aves desta família podem ser parasitadas por duas ou mais espécies do gênero Pteronyssoides.

Adultos e formas jovens do gênero Proctophyllodes, encontrado em nove espécies de aves Passeriformes (Tab. I), foram observados principalmente na face ventral das rêmiges e das retrizes. A análise estatística revelou que existem diferenças significativas entre os diferentes locais parasitados pelo gênero Proctophyllodes (Kruskall-Wallis $\left.\left(\mathrm{H}_{1}\right)=13,0936 ; \mathrm{p}<0,05\right)$. O teste de comparação múltipla de Kruskall-Wallis mostrou que a face ventral das rêmiges, diferiu significativamente dos demais locais parasitados, apresentando-se mais parasitado.

Estes ácaros, quando adultos, podem ser facilmente observados na pena, e segundo ATYEO \& BRAASCH (1966), tal fato se deve à pigmentação castanho-avermelhada da sua cutícula. De acordo com estes autores, o gênero Proctophyllodes é um dos mais ricos em número de espécies encontrados em aves, principalmente Passeriformes, sendo que apenas duas espécies, P. huitzilopochtlii Atyeo \& Braash, 1966 e P. scolopacinus (Koch, 1842), são ectoparasitos de aves não Passeriformes, das famílias Trochilidae e Scolopacidae, respectivamente. Estes mesmos autores citam, que os ácaros do gênero Proctophyllodes apresentam uma acentuada especificidade de hospedeiro, dos quais $80 \%$ dos ácaros por eles observados, apresentaram especificidade por determinadas famílias.

O gênero Pterodectes, coletado em dezoito hospedeiros (Tab. I), ocorreu principalmente na face ventral das rêmiges (em quinze hospedeiros), seguido da face ventral e dorsal das retrizes (em doze hospedeiros). Os locais parasitados por este gênero apresentam diferenças estatísticas significativas (Kruskall-Wallis $\left.\left(H_{1}\right)=29,0347, p<0,05\right)$. O teste de comparação múltipla de Kruskall-Wallis, revelou que todos os locais parasitados diferem entre si, sendo a face ventral das rêmiges o local mais parasitado. Segundo PARK \& ATYEO (1971), os hospedeiros principais são aves da ordem Passeriformes, apesar de que, oito, das vinte espécies descritas para este gênero ocorrem em aves da família Trochilidae (Apodiformes).

O gênero Trouessartia, presente em dezesseis espécies de hospedeiros, ocorreu preferencialmente na face ventral das rêmiges primárias (em sete hospedeiros), seguido da face dorsal das retrizes (em seis hospedeiros), sendo que em $S$. ruficollis, este gênero ocorreu nas duas faces, tanto nas rêmiges quanto nas retrizes (Tab. I). A análise dos dados revelou diferenças estatísticas significativas entre os locais parasitados pelo gênero Trouessartia (Kruskall-Wallis $\left(\mathrm{H}_{1}\right)=14,9026$; $\mathrm{p}<0,05)$. O teste de comparação múltipla de Kruskall-Wallis, mostrou que os locais parasitados diferem entre si, sendo a face ventral e dorsal das rêmiges, os locais mais parasitados. 
SANTANA (1976) cita que, os locais aonde este gênero é geralmente observado, são as rêmiges secundárias, as retrizes e as coberteiras das rêmiges e da cauda. SANTANA (1976) menciona ainda o gênero Trouessartia como ectoparasito restrito das ordens Coraciiformes (somente da Família Alcedinidae) e Passeriformes, apesar de existirem registros na literatura indicando associação com muitas aves não Passeriformes. RADFORD (1958) assinala este gênero para as ordens Pelecaniformes (Anhingidae e Fregatidae), Ciconiiformes (Threskiornithidae), Charadriiformes (Charadriidae), Coliiformes (Coliidae), Apodiformes (Apodidae) e nove famílias da ordem Passeriformes. BERLA (1960a) cita a espécie Trouessartia cactorum para um hospedeiro da família Psittacidae e GAUD \& ATYEO (1996) assinalam associações com as ordens Cuculiformes, Caprimulgiformes, Coraciiformes, Piciformes e principalmente, Passeriformes.

Para as andorinhas (Hirundinidae) foram descritas 22 espécies do gênero Trouessartia (GAUD \& ATYEO 1986), sendo dezessete provenientes de aves do Velho Mundo. Tanto SANTANA (1976), como GAUD \& ATYEO (1986), sugerem que estas aves podem abrigar simultaneamente mais de uma espécie deste gênero, porém, habitando microambientes diferenciados nas penas.

Na plumagem de $V$. jacarina (Emberizidae), encontrou-se cinco gêneros de ácaros plumícolas de diferentes famílias (Tab. I). ATYEO \& GAUD (1979) consideravam cinco, o número máximo de espécies de ácaros por indivíduo, até observarem um espécimem do beija-flor Ramphodon naevius (Dumont, 1818), com oito espécies diferentes.

Observou-se que os ácaros plumícolas adultos, estiveram dispostos nas rêmiges e retrizes, quase sempre com a região anterior do corpo voltada em direção à raque, paralelos às barbicelas das penas, presos pelas ventosas tarsais. Nas penas de contorno do corpo ou cabeça, os ácaros distribuíam-se por toda a porção plumácea das penas, enquanto que na porção penácea, os ácaros adotavam a mesma posição das rêmiges e retrizes.

Em vários hospedeiros, observou-se a presença de formas jovens de ácaros plumícolas na região da nuca e pescoço, provavelmente porque, na transferência dos ácaros, dos pais para os filhos, logo foi realizada a oviposição. ATYEO \& GAUD (1979) sugerem que a transferência de ácaros ocorra quando as penas de vôo dos filhotes estão 50 a $70 \%$ desenvolvidas e, aparentemente, os ácaros não apresentam preferência por lugar na pena, e à medida que as penas dos filhotes vão se desenvolvendo, os ácaros vão procurando uma localização preferencial.

No presente trabalho, observou-se taxas de prevalência que variaram de 3,2 a $100 \%$. Poucos são os relatos na literatura sobre a prevalência dos ácaros plumícolas. WHEELER \& THRELFALL (1986) assinalaram taxas que variaram de 80 a $100 \%$, em aves canadenses, MARINI et al. (1996) registraram de 66,7 a 100\% para aves da Floresta Atlântica e MARINI \& COUTO (1997), de 21 a 100\% em aves de florestas de Minas Gerais. PoULIN (1991) considera que a prevalência, quando baixa ou nula, pode indicar falta de exposição aos ectoparasitos e não à baixa susceptibilidade. Animais que vivem em grupos apresentam uma maior chance de adquirir e acumular ectoparasitos devido a grande proximidade e número de contatos físicos entre os membros dos grupos, do que aqueles que apresentam vida solitária, menciona POULIN (1991). 


\section{CONCLUSÕES}

Dentre os ácaros identificados no presente trabalho, a família Proctophyllodidae foi a que apresentou o maior número de hospedeiros. O gênero Pterodectes (Pterodectinae) ocorreu no maior número de hospedeiros $(\mathrm{n}=18)$, seguido dos gêneros Trouessartia (Allodectinae) $(\mathrm{n}=16)$ e Proctophyllodes (Proctophyllodinae) $(n=9)$.

No que se refere à localização, verificou-se que alguns grupos de ácaros apresentaram preferências por determinados locais no corpo das aves, principalmente pelas rêmiges e retrizes. E nestas penas parasitavam principalmente a face ventral. As regiões menos parasitadas foram a cabeça, o uropígeo e as penas supra-caudais.

AGRADECIMENTOS. Agradecemos à CAPES pela bolsa de mestrado; ao Curso de Mestrado em Biologia Animal da Universidade Federal de Pernambuco; ao auxílio em campo de Gilmar Farias, Fabrícia Leal, Roberta Rodrigues e Rossano Mendes Pontes; ao Prof. Galileu Coelho (UFPE) pela co-orientação deste trabalho; ao Dr. João Luiz Horácio Faccini (UFRRJ) pela ajuda na identificação dos ácaros plumícolas, e aos dois revisores anônimos pelas valiosas sugestões.

\section{REFERÊNCIAS BIBLIOGRÁFICAS}

Atyeo, W.T. \& N.L. BraAsch. 1966. The feather mite genus Proctophyllodes (Sarcoptiformes: Proctophyllodidae). Bull. Univ. Nebraska St. Mus. 5: 1-354. AtYEO, W.T. \& J. GAUD. 1979. Feather mites and their hosts. p. 355-361. In: J.G. RodrigueZ (Ed.). Recent Advances in Acarology II. New York, Academic Press, 530p.

455-458.

BERLA, H.F. 1958a. Analgesidae Neotropicais. I - Duas novas espécies de Pterodectes Robin, 1868 (Acarina - Proctophyllodinae) coletadas em Fringillidae, Aves, Passeriformes. Bol. Mus. Nac. (Zool.), Rio de Janeiro, 186: $1-6$.

1958b. Analgesidae Neotropicais. III - Redescrição de Pteronyssus chiasma Trouessart, 1885 (Acarina, Pterolichinae), hóspede de Rhamphastidae (Aves, Piciformes). Rev. Brasil. Biol. 18 (3): 337-339.

— 1959a. Analgesoidea Neotropicais. V - Sobre uma espécie nova de Proctophyllodes Robin, 1868 e redescrição de Pterolichus varians selenurus Trouessart, 1898 (Acarina, Pterolichinae). Rev. Brasil. Biol. 19 (2): 203-206.

Trouessartia Canestrini, 1889 (Acarina-Proctophyllodidade), hóspedes de Fringillidae (Aves, Passeriformes). Bol. Mus. Nac. (Zool.), Rio de Janeiro, 208: $1-8$.

1959c. Analgesoidea Neotropicais. IV - Sobre algumas espécies novas pouco conhecidas de acarinos plumícolas. Bol. Mus. Nac. (Zool.), Rio de Janeiro, 209: 1-17.

—. 1959d. Analgesoidea neotropicais. VI - Um novogênero de acarinos plumícolas (Acarina, Proctophyllodidae), hóspede de Oxyruncidae (Aves, 
Passeriformes). Stud. Entomol. 2: 31-32.

1960a. Analgesoidea Neotropicais. VII - Novas espécies de acarinos plumícolas. An. Acad. Bras. Ciênc. 32 (1): 95- 105.

. 1960b. Analgesoidea Neotropicais. VIII - Acarinos plumícolas parasitas de aves do Brasil. Rev. Brasil. Biol. 20 (2): 149-153.

1962. Analgesoidea Neotropicais. IX - Uma nova espécie de Trouessartia Canestrini, 1899 (Acarina-Proctophyllodidae). Bol. Mus. Nac. (Zool.), Rio de Janeiro, 241: 1-5.

FACCINI, J.L.H.; J. GAUD \& W.T. ATYEO. 1976. Descrição de Eurydiscalges g.n. (Analgidae, Sarcoptiformes), com quatro espécies novas parasitas de Psittacidae (Aves), provenientes da América do Sul. Rev. Brasil. Biol. 36 (3): 701-707.

FACCINI, J.L.H. \& W.T. ATYEO. 1981. Generic revisions of the Pteronyssinae and Hyonyssinae (Analgoidea: Avenzoariidae). Proc. Acad. Nat. Sci. Philadelphia 133: $20-72$.

FlechtManN, C.H.W. 1975. Elementos de Acarologia. São Paulo, Nobel, 344p. 1981. New records of mites from Brazil, with description of two new species in the genus Oligonychus Berlese (Acari, Tetranychidae). Rev. Brasil. Biol. 41 (4): 861-866.

GAuD, J. \& W.T. ATYEO. 1977. Nouvelles superfamilles pour les acariens astigmates parasites d'oiseaux. Acarologia 19 (4): 678-685.

- 1979. Co-évolution des acariens sarcoptiformes plumicoles et de leurs hôtes. Acarologia 21 (3): 291-306.

1985. Les acariens du genre Pteronyssoides (Avenzoariidae, Analgoidea) parasites des hirondelles euroafricaines. Acarologia 26 (3): 295-306.

- 1986. Les Trouessartia (Analgoidea, Trouessartiidae) parasites des hirondelles de L'ancien monde. I - Le group appendiculata. Acarologia 27 (3): 263-274.

1996. Feather mites of the world (Acarina, Astigmata): the supraspecific taxa. Part I. Ann. Zool. Wetenschappen 277: 1-187.

Margolis, L.; G.W. EsCH; J.C. Holmes; A.M. KurIs \& G.A. SCHAD. 1982. The use of ecological terms in parasitology (Reported of an ad hoc Committee of the American Society of Parasitologists). Jour. Parasitol. 68 (1): 131-133.

Marini, M.A.; B.L. ReInerT; M.R. Bornschein; J.C. PInTO \& M.A. Pichorim. 1996. Ecological correlates of ectoparasitism on Atlantic Forest birds, Brazil. Ararajuba 4 (2): 93-102.

MARINI, M.A. \& D. COUTO. 1997. Correlações ecológicas entre ectoparasitas e aves de florestas de Minas Gerais. p. 210-218. In: L.L. LeITE \& C.H. SAITo (Eds). Contribuição ao Conhecimento Ecológico do Cerrado - Trabalhos Selecionados do $3^{\circ}$ Congresso de Ecologia do Brasil. Brasília, Departamento de Ecologia, Universidade de Brasília, 323p.

NovaES, F.C. 1953. A new species of Neumanniella from the Tataupa tinamou (Sarcoptiformes, Analgesidae). Rev. Brasil. Biol. 13 (2): 203-204.

Novaes, F.C. \& J.C. Carvalho. 1952a. A new genus and species of feather mite (Pterolichinae, Analgesidae. Rev. Brasil. Biol. 12 (2): 211-214.

1952b. A new species of Megninia from the roseate spoonbill (Analgesidae, Analgesinae). An. Acad. Bras. Ciên. 24 (3): 303-306. 
PARK, C.K. \& W.T. ATYEO. 1971. A generic revision of the Pterodectinae, a new subfamily of feather mites (Sarcoptiformes: Analgoidea). Bull. Univ. Nebraska St. Mus. 9 (3): 39-88.

PoulIN, R. 1991. Group-living and infestation by ectoparasites in passerines. Condor 43: 418-423.

RADFORD, C.D. 1958. The host-parasite relationship of the feather mites (Acarina: Analgesoidea). Revta bras. Ent. 8: 107-170.

SANTANA, F.J. 1976. A review of the genus Trouessartia (Analgoidea: Alloptidae). Jour. Med. Ent. Suppl. 1: 1-128.

WHEELER, T.A.\& W. THRELFALl. 1986. Observations on the ectoparasites of some Newfoundland passerines (Aves: Passeriformes). Can. Jour. Zool. 64: 630-636.

Recebido em 27.II.1998; aceito em 27.VIII. 1999. 